\title{
Clinical Toxicology
}

\section{Strychnine poisoning as an unusual cause of convulsions}

\author{
D.J. Burn' ${ }^{1}$ C.R.V. Tomson ${ }^{1}$, J. Seviour ${ }^{2}$ and G. Dale ${ }^{2}$
}

${ }^{1}$ Department of Medicine, Royal Victoria Infirmary, Newcastle upon Tyne and ${ }^{2}$ Department of Clinical
Biochemistry, Newcastle General Hospital, Newcastle upon Tyne, UK.

Summary: A fatal case of strychnine poisoning is presented. The patient vomited then suffered a series of tonic convulsions which were triggered by tactile stimulation. In between paroxysms he was initially alert. Eventually the patient became comatosed due to anoxia and had a cardiac arrest. He presented with a marked metabolic acidosis and rapidly developed renal failure caused by acute rhabdomyolysis. This clinical picture is classical for strychnine poisoning and the complications which the intoxication produces. Attention is drawn to the fact that survival can even follow the ingestion of very large doses of strychnine providing there is no delay in diagnosis and treatment.

\section{Introduction}

Strychnine ingestion is an uncommon and a peculiarly unpleasant form of poisoning. ${ }^{1}$ The analeptic nature and mechanism of action of the alkaloid has long been recognized. ${ }^{2}$ Although low in the differential of causes of convulsions the classical clinical picture of strychnine poisoning is important to recognize because with early and appropriate treatment survival can follow the ingestion of even very large doses. ${ }^{3}$ We present the case of a farmer who died from strychnine poisoning. Although we cannot deduce from our case that early diagnosis and intervention may be life-saving, this has been well documented. ${ }^{4}$ We feel, however, that our case graphically illustrates the presentation of strychnine poisoning.

\section{Case report}

A 32 year old farmer was admitted to this hospital in acute renal failure of unknown aetiology. The history, obtained from his wife and ambulance crew, was that he had woken his wife at $05.00 \mathrm{~h}$ telling her that their nearby barn was on fire. The couple removed farm equipment from the barn but his wife later denied any possibility of smoke or fume inhalation. Thirty minutes later, while waiting for the fire brigade, the farmer vomited and after a few minutes had a series of tonic convulsions. Between convulsions he was alert and calling for help, but any physical contact appeared to cause pain, panic and further convulsions. After several paroxysms of convulsions he became cyanosed

Correspondence: D.J. Burn, M.A., M.R.C.P., Department of Neurology, Royal Victoria Infirmary, Queen Victoria Road, Newcastle upon Tyne NE1 4LP, UK.

Accepted: 24 February 1989 and lost consciousness. On arrival of the fire brigade and ambulance services no pulses were palpable and cardiopulmonary resuscitation was given until an output was restored. On arrival at the local hospital he was no longer cyanosed and appeared well perfused with a sinus tachycardia. Further convulsions were controlled with intravenous diazepam, paralysis and mechanical ventilation. Initial investigations performed some 15 minutes after restoration of a cardiac output, and as the patient was being intubated, revealed metabolic acidosis $\left(\mathrm{pH} \mathrm{6.6} ; \mathrm{PCO}_{2}=7.91\right.$ $\mathrm{kPa}, \mathrm{PO}_{2}=38.2 \mathrm{kPa}$ on $100 \%$ oxygen by mask) and a plasma creatinine of $130 \mu \mathrm{mol} / \mathrm{l}$. Despite the correction of the acidosis with $200 \mathrm{ml}$ of $8.4 \%$ intravenous sodium bicarbonate and maintenance of an adequate blood pressure (systolic blood pressure $>130 \mathrm{mmHg}$ ) the urine output remained less than $15 \mathrm{ml} / \mathrm{h}$. He was transferred to this hospital for dialysis, with a presumptive diagnosis of carbon monoxide poisoning.

Investigations on admission: plasma creatinine = $410 \mu \mathrm{mol} / \mathrm{l}$; creatine kinase $>1500$ units per litre (normal less than 175 units per litre); carboxyhaemoglobin level $2 \%$.

Microscopic crystals found in the urine raised the question of oxaluria secondary to ethylene glycol poisoning - for which treatment was instituted. However, gas chromatography, performed on a 1 foot column packed with $0.8 \%$ THEED on carbopack $C$ at $110^{\circ} \mathrm{C}$, indicated that ethylene glycol was not present in the plasma. Furthermore, X-ray diffraction studies revealed that the crystals were not oxalate - but remained unidentified. In view of this, further toxicological investigations were carried out on the urine. In addition to a benzodiazepine presumably administered after admission - thin layer chromato- 
graphy using the 'Toxi Lab' chromatography system (Mercia Laboratories) showed the presence of a well-marked compound with migration and staining characteristics of strychnine. This was confirmed by gas chromatography/mass spectrometry using an HP Ultra 2 column, temperature programmed from $70-300^{\circ} \mathrm{C}$ in a Hewlett Packard gas chromatography (HP 5890) coupled to a HP 5970 bench top mass spectrometer. This showed the presence of a compound which eluted from the column in 23 minutes and had a characteristic mass spectrum with a base peak at $\mathrm{m} / \mathrm{z} 334$ and other prominent peaks including those at 162 and 120 which matched the published spectrum for strychnine and corresponded to that stored in the data base of the computer interfaced to the system. In addition, an authentic sample of strychnine was shown to have the same characteristic retention time and mass spectrum. Using the same technique, strychnine was also shown to be present in plasma but quantitation was not carried out.

Despite regular dialysis and continued ventilation the patient remained in a vegetative state and died six days later. Close questioning of the relatives revealed a history of marital conflict and financial worries. A coroner's inquiry returned an open verdict.

\section{Discussion}

Strychnine is an alkaloid found in the seeds of Strychnos nuxvomica, a vine of India. It is used as a rodenticide and pesticide but is also employed in the illicit manufacture of some narcotics, notably cocaine. ${ }^{4}$ Strychnine and its salts have a bitter taste when dissolved in water.

The alkaloid exerts an excitatory effect on the central nervous system by preventing glycine uptake at inhibitory synapses, mainly in the ventral horns of the spinal cord. This explains why minimal peripheral sensory stimulation is needed to trigger diffuse muscle

\section{References}

1. Norris, C.J. Corn, whiskey and strychnine poisoning. Am J Clin Pathol 1937, 7: 531-535.

2. Olmsted, J.M.D. Francois Magendie. Pioneer in Experimental Physiology and Scientific Medicine in XIX Century France. Schuman, New York, 1944.

3. Perper, J.A. Fatal strychnine poisoning: a case report and review of the literature. $J$ Forensic $S c i$ 1985, 30: $1248-1255$.

4. Edmunds, M., Sheenan, T.M.T. \& Van't Hoff, W. Strychnine poisoning: clinical and toxicological observations on a non-fatal case. Clin Toxicol 1986, 24: 245-255.

5. Winek, C.L., Wahba, W.W., Esposito, F,M. \& Collom, W.D. Fatal strychnine ingestion. J Anal Toxicol 1986, 10: $120-121$. contractions. ${ }^{5}$ Strychnine also has an excitatory action on the medulla and, curiously, enhances the sensations of touch, smell, hearing and sight. ${ }^{6}$ Other reports of strychnine poisonings have noted vomiting, hyperthermia and horizontal pendular nystagmus ${ }^{7}$ as clinical manifestations. Our case was thus typical, with vomiting and hypersusceptibility to external stimuli so that any attempts to help the farmer caused pain and further convulsions.

Strychnine is readily absorbed after oral administration. It is thought to be metabolized mainly in the liver, with a plasma half-life of 10 hours $^{4}$ although up to $20 \%$ of a dose is excreted unchanged in the urine. ${ }^{8}$

Fatal doses of strychnine are reported to be as low as $5-10 \mathrm{mg}^{5,8}$ but, more significantly, survival can follow ingestion of very high doses (over $3500 \mathrm{mg}$ ). ${ }^{3}$ If recognized in time, symptomatic treatment with diazepam or barbiturates to control the convulsions and paralysis and mechanical ventilation where necessary can prevent death from anoxia ${ }^{9}$ or from the associated complications of lactic acidosis, rhabdomyolysis and acute renal failure. ${ }^{4}$ In our case, acute renal failure developed rapidly and was presumed to be secondary to rhabdomyolysis. Death, however, was primarily due to anoxic brain damage.

We conclude that strychnine ingestion should be considered in all cases of convulsion of obscure aetiology, especially if the person is an agricultural worker or is known to have access to the poison. If available, a carefully taken history from a witness may help make the diagnosis because of the classical clinical picture of strychnine poisoning. Prompt and adequate anticonvulsant treatment followed by effective artificial ventilation can be life-saving.

\section{Acknowledgements}

We are grateful to Dr Katherine Liddell for performing the $\mathrm{X}$-ray studies on urinary crystals and to Miss Bernadette McNestry for preparing the manuscript.

6. Burgen, A.S.V. \& Mitchell, J.F. (eds) Gaddum's Pharmacology, VIII edition. Oxford Medical Publications, Oxford, 1978, pp. 62-63.

7. Blain, P.G., Nightingale, S. \& Stoddart, J.C. Strychnine poisoning: abnormal eye movements. Clin Toxicol 1982 , 19: $215-217$.

8. Clarke, E.G.C. Isolation and Identification of Drugs in Pharmaceuticals, Body Fluids and Post-Mortem Material, 2nd edition. Pharmaceutical Press, London, pp. 976-977.

9. Hawkins, G.F. Two cases of strychnine poisoning in children. Br Med J 1962, ii: 26. 